\title{
A STUDY OF THE PERFORMANCE OF THE MODIFIED TRANS- FORMED VEGETATION INDEX MTVI
}

\author{
Skianis G. Aim. ${ }^{1}$, Gournelos Th. ${ }^{1}$, Vaiopoulos D. ${ }^{1}$, and Nikolakopoulos K. ${ }^{2}$ \\ ${ }^{1}$ University of Athens, Faculty of Geology and Geoenvironment, Department of Geography and Climatol- \\ ogy,15784 Athens, Greece, skianis@geol.uoa.gr,gournelos@geol.uoa.gr,vaiopoulos@geol.uoa.gr \\ ${ }^{2}$ Institute of Geological and Mineral Exploration (IGME), Olympic Village Entrance C, Aharnae 13 671, \\ Greece,knikolakopoulos@igme.gr
}

\begin{abstract}
In the context of a recent research on the performance of vegetation indices we have shown, with the aid of probability theory, that the shape and width of the histogram of the Transformed Vegetation Index TVI is controlled by the ratio of the standard deviation of the Red band to that of the NIR band. Therefore a modification of the mathematical expression of the TVI vegetation index may produce images with a varying tonality contrast. In the present paper the modified transformed vegetation index MTVI is introduced, the value of which is controlled by a positive parameter $c$. A theoretical study of the effect of this parameter on the image histogram is first carried out and it is shown that changing c one can obtain MTVI images with different histograms and standard deviations. Experimentation with a satellite image over western Peloponnese verifies that the parameter c controls the shape of the MTVI histogram and, furthermore, the optical effect of the MTVI image as well as the spatial variation (semivariogram) of the pixel values. Therefore the proposed modified transformed vegetation index may help the potential user in broadening his/her choices to map the vegetation cover of the area under study.
\end{abstract}

Key words: vegetation index, TVI, MTVI, parameter c, standard deviation, semivariogram.

\section{Introduction}

The mapping of the land cover is essential for local and regional planning. Furthermore, studying the role of terrestrial vegetation is crucial in understanding how the earth functions as a system. A measure of the vegetation cover and green biomass may be obtained by calculating the vegetation index at each pixel of a multispectral image.

An abundance of vegetation indices have been proposed, in order to map vegetation cover. A comprehensive list of these indices is made by Jensen (2000). Most of the vegetation indices have been introduced and evaluated taking into account empirical criteria of response over land cover types of interest (Gitelson 2004, Malinis et al. 2004, Kale 2005, Silleos et al. 2006). Mathematical models which associate the vegetation cover with its reflectance at various spectral bands have also been developed (Goel 2008, Haboudaine et al. 2004). A probabilistic approach on the problem of the performance of a vegetation index has been proposed by Vaiopoulos et. al. 2004.

The transformed vegetation index TVI has been introduced by Deering et al. 1975. It has a particular interest, since it produces images with a strong tonality contrast, which may help in detecting burnt areas and soils with a poor vegetation (Skianis et al. 2007a, b). 


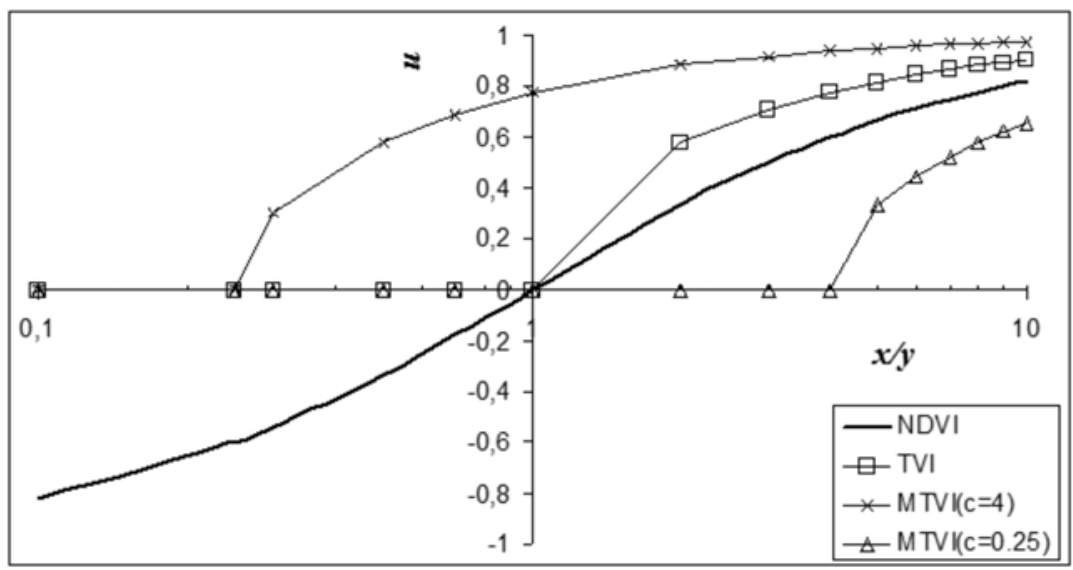

Fig. 1: NDVI, TVI and MTVI variation against $x / y$.

The TVI is defined by (Deering et al. 1975):

$$
u=\left\{\begin{array}{cc}
\sqrt{\frac{x-y}{x+y}} & \text { for } x \geq y \\
0 & \text { for } x<y
\end{array}\right.
$$

$u$ is the value of the vegetation index $x$ and $y$ are the tonalities or reflectances of the Near Infrared and Red bands, respectively. This vegetation index may be alternatively expressed as $\operatorname{sqrt}[(x-y) /(x+y)$ +0.5 ] in order to avoid negative values of the quantity under the square root, but in the present paper the definition of the TVI according to relation (1) is adopted.

Skianis et al. (2007b) have shown that modifying the expression for the TVI may produce images with a varying optical effect, which may be useful in recognizing targets of interest and mapping the land cover. The modified transformed vegetation index MTVI is defined by (Skianis et al. 2007b):

$$
u=\sqrt{\frac{c x-y}{c x+y}} \text { for } c x>y \quad(u=0 \text { if } c x £ y)
$$

$c$ is a parameter which takes values between 0.1 and 10 and controls the tonality contrast of the image.

In Fig. 1 the variation of TVI and MTVI against $x / y$ is presented, according to the relations (1) and (2). The behavior of the frequently used NDVI vegetation index, which has been introduced by Rouse et al. (1973) and is defined by $u=(x-y) /(x+y)$ is also presented. It can be observed that the MTVI takes higher values as long as $c$ increases.

In this paper the statistical behaviour as well as the optical effect of the MTVI image is studied. First a mathematical expression for the MTVI histogram is derived, according to a probabilistic approach which has been developed by Vaiopoulos et al. (2004). The standard deviation is then calculated for various values of the $c$, in order to have a quantitative measure of the variation of the tonality contrast with this parameter. Then, the MTVI is applied on an ASTER image of the area of Olympia (western Peloponnese) in order to assess the optical effect and study the spatial variation of the produced MTVI images. 


\section{The MTVI histogram, according to the probabilistic approach}

In order to study the statistical behaviour of the MTVI vegetation index, the histograms of the NIR and Red bands, $\mathrm{x}$ and $\mathrm{y}$ respectively, have to be simulated by a proper distribution. It is reasonable to assume that this distribution is zero for a null brightness value, presents a peak at a relatively low value of the tonality range and gets practically nullified at high brightness values. In practice, the histogram may be more complicated and present more than one peaks, but it is reasonable to be approximated by a simple distribution, which may help the mathematical analysis.

Vaiopoulos et al. 2004 have proposed distributions $p$ of the form $p(x)$ (or $p(y)) \sim x \cdot \exp \left(-a x^{2}\right)$ (or $p(y)$ $\sim y$.exp $\left(-a y^{2}\right)$ ), in order to describe the histograms of $x$ and $y$ bands. In the same paper, it has been derived the expression for the distribution $g\left(u^{\prime}\right)$ of the values $u^{\prime}$ of the MNDVI vegetation index, which is defined by (Vaiopoulos et al. 2004):

$$
u^{\prime}=\frac{c x-y}{c x+y}
$$

$c$ is the same parameter which appears in relation (2) for the MTVI vegetation index.

According to Vaiopoulos et al. 2004, $g\left(u^{\prime}\right)$ is given by:

$$
g\left(u^{\prime}\right)=\frac{4 \lambda c^{2}\left(1-u^{\prime 2}\right)}{\left[\lambda\left(1+u^{\prime}\right)^{2}+c^{2}\left(1-u^{\prime}\right)^{2}\right]^{2}}
$$

$\lambda$ is given by (Skianis et al. 2007a):

$$
\lambda=\left(\frac{\operatorname{stdev}(y)}{\operatorname{stdev}(x)}\right)^{2}
$$

The $u$ values of the MTVI are is related to the $u^{\prime}$ values of the MNDVI, by $u^{\prime}=u^{2}$, for $u^{\prime} \geq 0$ (or $c x$ $\geq y$ ). According to Spiegel 1977, the distribution $g(u)$ of the MTVI values is related to $g(u$ ') by:

$$
g(u)=g\left[u^{\prime}(u)\right] \cdot\left|\frac{d u^{\prime}}{d u}\right|
$$

Combining the relations (4) and (6) and taking into account that $d u^{\prime} / d u$ gives:

$$
g(u)=\frac{8 \lambda c^{2}\left(1-u^{2}\right) u}{\left(1+u^{2}\right)^{3}\left[\lambda+c^{2}\left(\frac{1-u^{2}}{1+u^{2}}\right)^{2}\right]^{2}} \quad \text { for } u^{\prime} \geq 0(\text { or } c x \geq y)
$$

For $u^{\prime}<0$, or $c x<y, u$ is equal to zero, according to relation (2). The percentage $g(0)$ of the null TVI values, is given by:

$g(0)=\int_{-1}^{0} g\left(u^{\prime}\right) d u^{\prime}$

Combining relations (4) and (8) gives: 

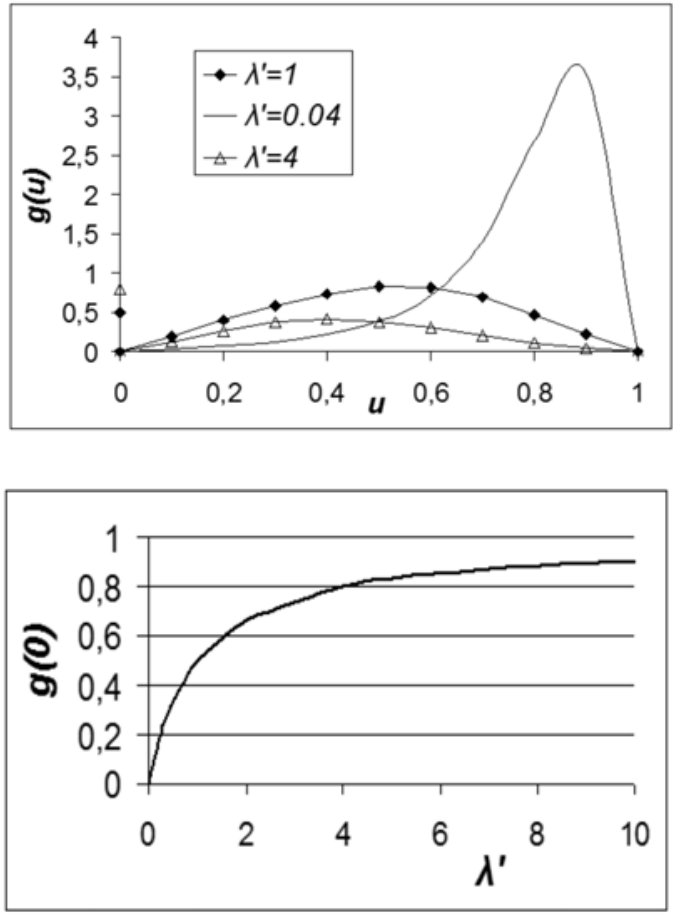

Fig. 2: The distribution $g(u)$ of the MTVI values, for various $\lambda$ ' values.

Fig. 3: The percentage $g(0)$ of the zero MTVI values against $\lambda$.

$$
g(0)=\frac{\lambda}{\lambda+c^{2}}
$$

For convenience in the further statistical treatment, the quantity $\lambda$ ' is introduced, which is defined by:

$$
\lambda^{\prime}=\lambda / c^{2}
$$

According to relations (7), (9) and (10), the expression for $g(u)$, which describes the histogram of the MTVI image, is:

$$
g(u)= \begin{cases}g(0)=\frac{\lambda^{\prime}}{\lambda^{\prime}+1} & \text { for } c x<y \\ \frac{8 \lambda^{\prime}\left(1-u^{2}\right) u}{\left(1+u^{2}\right)^{3}\left[\lambda^{\prime}+\left(\frac{1-u^{2}}{1+u^{2}}\right)^{2}\right]^{2}} & \text { for } c x \geq y\end{cases}
$$

There is a remarkable similarity between the expression for the distribution $g(u)$ of the MTVI values (relation (11)) and that of the TVI values (Skianis et. al. 2007a). The only difference is that in the former the parameter $\lambda$ ' appears, while in the latter parameter $\lambda$ appears instead.

In Fig. 2 the distribution $g(u)$ of the MTVI values for various $\lambda$ ' values is presented. As long as $\lambda$ ' decreases (which means that $c$ increases) the peak of the distribution is shifted to the right. On the other hand, as it can be seen in Fig. 3, as long as $c$ increases ( $\lambda$ ' decreases), the percentage $g(0)$ of 


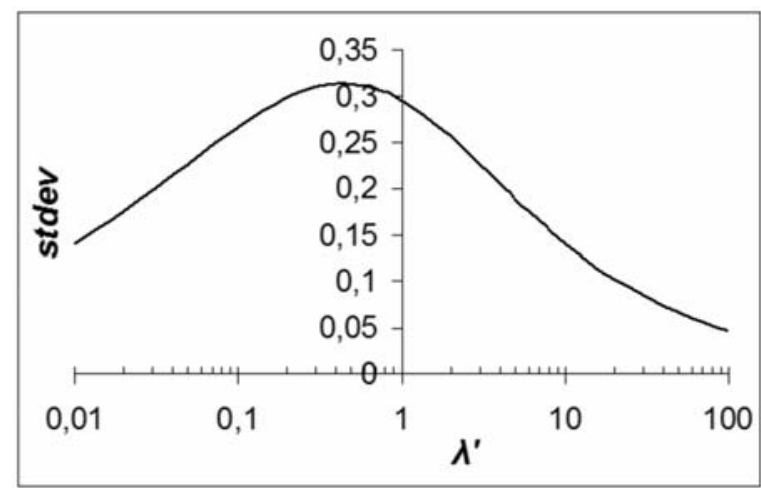

Fig. 4: The stdev variation of the MTVI values against $\lambda$ '.

the null values -in other words the percentage of the image pixels with null tonality- decreases. Therefore for high $c$ values the MTVI image is expected to be bright. Low $c$ values are expected to produce dark images.

The standard deviation stdev of $g(u)$, which describes the MTVI image histogram, was numerically calculated for various $\lambda$ ' values. In Fig. 4 , the stdev variation against $\lambda$ ' is presented. It can be ob-

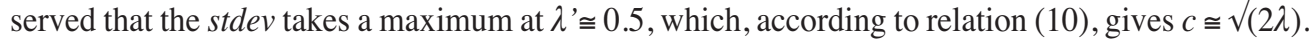
For this value of $c$ the MTVI image is expected to have a maximum standard deviation, therefore the strongest tonality contrast.

The probabilistic approach gave a picture of how the parameter $c$ influences the optical effect and statistical behavior of the MTVI image. Theoretical predictions have to be tested with real data, obtained by satellite images.

\section{Experimentation with a satellite image}

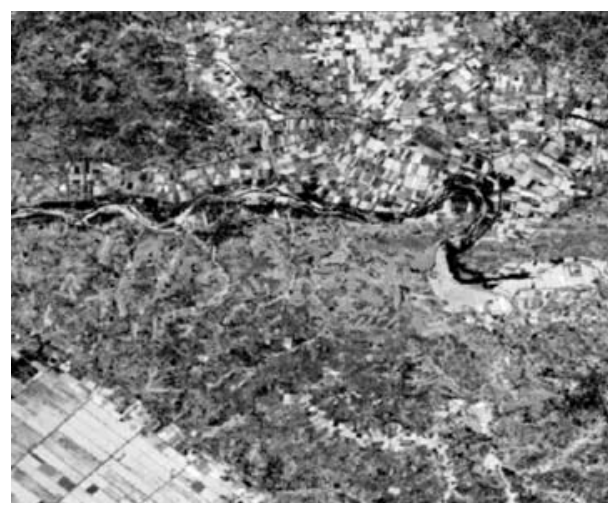

Fig. 5: A TVI ASTER image of a part of Western Peloponnese around Alfios river.

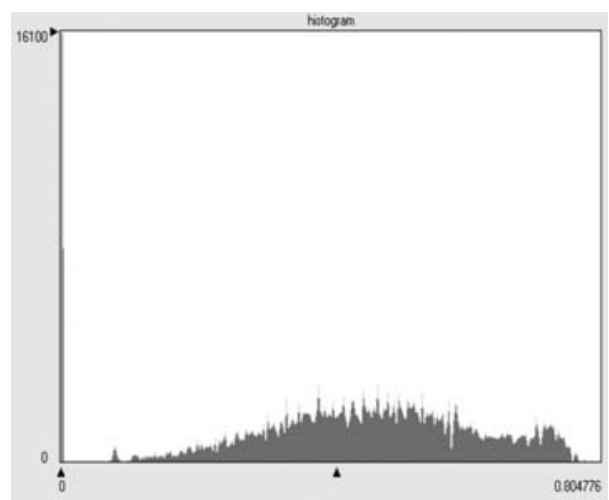

Fig. 6: The histogram of the TVI image.

The MTVI vegetation index, for various $c$ values, was applied in a satellite ASTER image of July 2008 , around the region of Olympia (western Peloponnese). The ratio $\lambda$ of the satellite image was found to be equal to 1.006. The MTVI images where produced using ERDAS Imagine software. A special tool to calculate the MTVI values was developed, using the spatial modeller of this software. In Fig.5, the TVI image $(c=1)$ of the area is presented. Alfios river is expressed with a curved 
line at the middle of the image, with direction East West. At the upper left and upper right part of the image the tonality is quite dark and expresses burnt areas (the fire took place one year before the acquisition of the image). Burnt areas with dark tonalities are also present at the middle of the image, below the river. Areas with dense vegetation and agricultural land are expressed with bright tones. In Fig. 6, the histogram of the image is presented. A great number of pixels have a null tonality and the others take values up to 0.885 .

In Fig. 7 the MTVI image of the same area, for $c=0.5$, is presented. It can be observed that the image has an overall dark tonality, which is also expressed in the image histogram of Fig. 8. The histogram is apparently blank because most pixels have null or very small tonalities. Therefore a small $c$ value (less than unity) produces an MTVI image with dark tones and $u$ values accumulated at zero point.

In Fig. 9 the MTVI image for a high $c$ value equal to 4 is presented. The image is considerably brighter than those of Figs 5 and 7 and the MTVI histogram of Fig. 10 is shifted to the right.

The observation of the TVI and MTVI images shows that there is an accordance, in qualitative terms, between theoretical predictions and real data. Low $c$ values produce dark images and histograms shifted to low $u$ values, while high $c$ values produce bright images with histograms shifted towards high $u$ values.
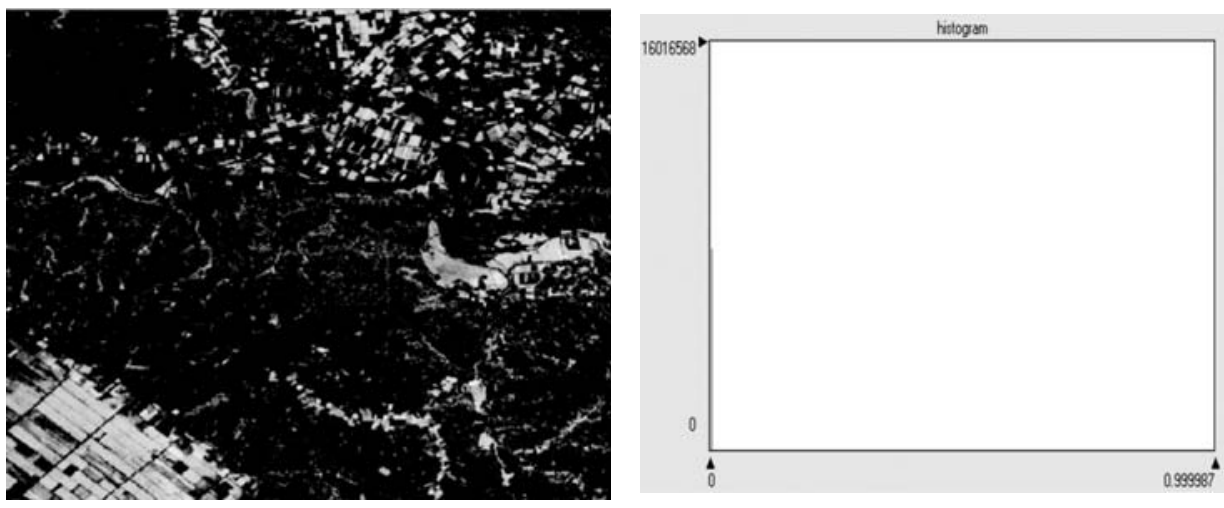

Fig. 7: The MTVI ASTER image for $c=0.5$.

Fig. 8: The MTVI histogram for $c=0.5$.
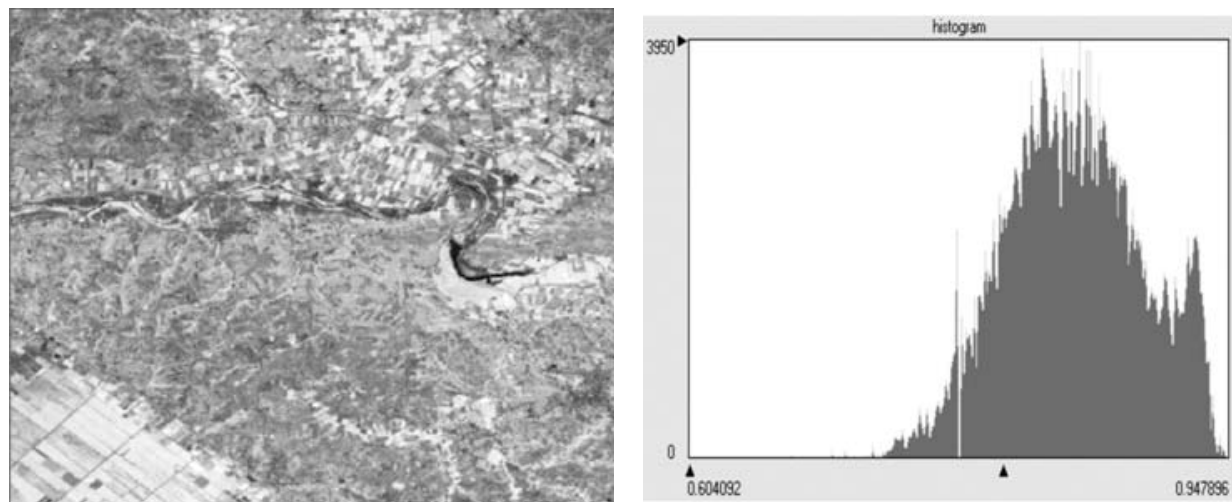

Fig. 9: The MTVI ASTER image for $c=4$.

Fig. 10: The MTVI histogram for $c=4$.

No histogram stretch was applied on the images of Figs 5, 7 and 9. The dark tonality of the MTVI image for $c=0.5$ (Fig. 5) does not favour the different land cover types to be expressed clearly. If a 


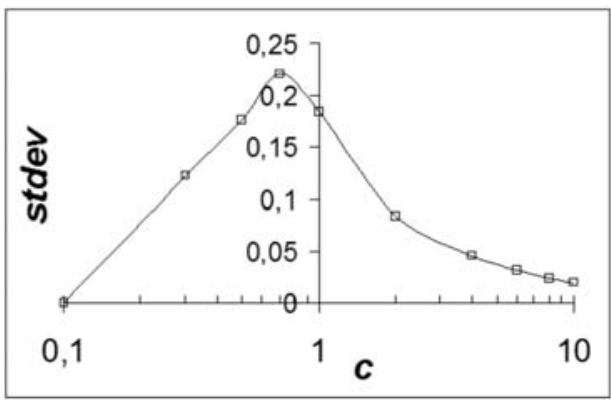

Fig. 11: The standard deviation of the MTVI ASTER images of part of western Peloponnese against $c$.

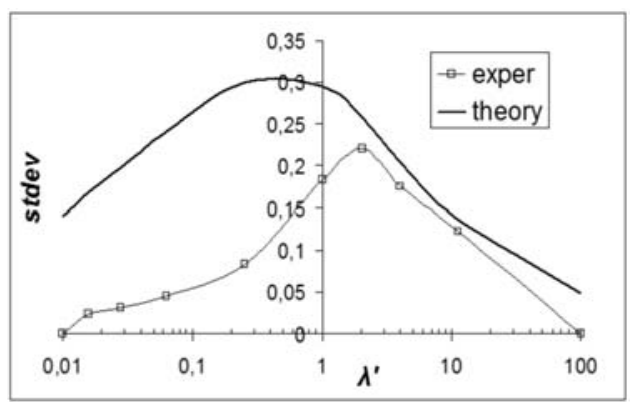

Fig. 12: Theoretically predicted and actual standard deviation values of the MTVI ASTER images against $\lambda$.

linear histogram stretch is applied on this image, the vegetation cover, as well as the river zone, are expressed with very bright tonalities, in contrast to the dark tonality of the other formations.

In the MTVI image for $c=4$ (Fig. 9) the eastern part of Alfios river is expressed with a dark tonality which is considerably lower than that of the other parts of the river. Particularly this tonality contrast is not so strong in the TVI image (Fig. 5). Actually a high $c$ value (more than unity) produces images with a diversification in the tonalities of dark areas. Therefore the parameter $c$ controls the contrast of the MTVI image, as well as the optical effect by which the various land cover types are expressed.

In order to study in quantitative terms the performance of the MTVI vegetation index on the ASTER image, the standard deviation (stdev) of the MTVI histogram for each $c$ was calculated. In Fig. 11 the variation of the stdev of the produced MTVI images against $c$ is presented. It can be observed that the stdev takes its maximum value for $c=0.7$. According to the theoretical predictions, the peak should be at $c=\sqrt{ }(2 \lambda)=1.4$ (since the $\lambda$ value of the ASTER image is equal to unity). Actually, in quantitative terms, there are deviations between theoretically predicted and actual stdev values, as it can be seen in Fig. 12. The differences are smaller for high $\lambda^{\prime}\left(=\lambda / c^{2}\right)$ values and bigger for low $\lambda^{\prime}$ values.

The deviations between theory and actual data should be attributed to the assumptions upon which the probabilistic approach is based. The assumptions are: a) to describe the histograms of the NIR and Red bands can be described by simple distributions $\left.p(x) \sim x \cdot \exp \left(-a x^{2}\right), b\right)$ the reflectances of the NIR and Red bands are independent each other (Vaiopoulos et al. 2004). Actual histograms are more complicated than the distribution $p$ and a considerable correlation between NIR and Red values exists.

On the other hand there are similarities in the behaviour of the theoretical and actual stdev curves of Fig. 12. Both curves present a maximum around $\lambda^{\prime}=1$ and relatively high (low) actual stdev values correspond to high (low) theoretically predicted ones.

As a general comment, it can be stated that the probabilistic approach and the experimentation with the ASTER image showed that the parameter $c$ in the expression for the MTVI tunes the optical effect and the standard deviation (in other words the tonality contrast) of the MTVI image.

Since the standard deviation is only a measure of the width of the contrast and does not provide any direct information about the spatial variation of the MTVI values, it is important to study this issue using a proper geostatistical function. 


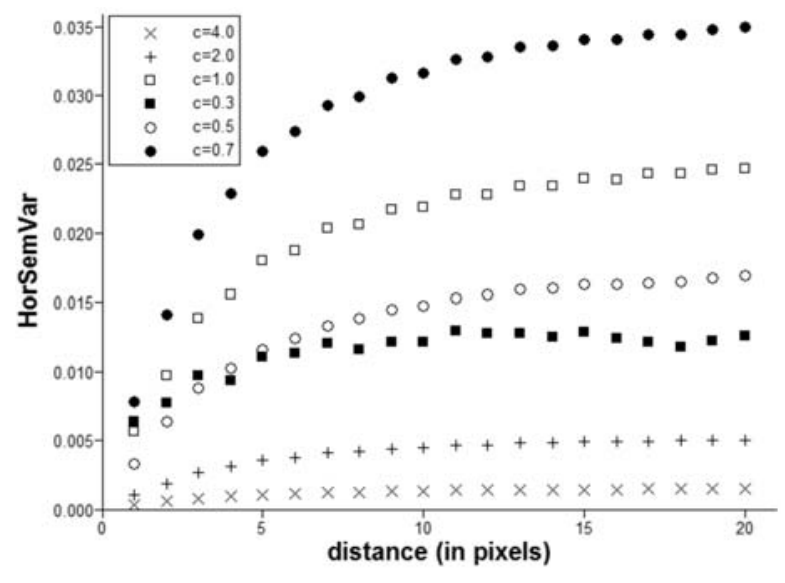

Fig. 13: The horizontal semivariograms of MTVI images for different $c$ values.

\section{The spatial variation of the MTVI images}

The semivariogram of a digital image represents the variance of the tonality differences between pixels at various distances each other, therefore it provides information of the spatial variation of the tonality. The semivariograms of the MTVI images, for different $c$ values were calculated, in order to see how the MTVI values vary in space. The ILWIS software was used for this purpose.

In Fig. 13 the horizontal semivariograms of MTVI images, for different values of $c$, are shown. The vertical semivariograms are not presented, since they have the same behaviour and small differences in the values of the semivariogram functions. It can be observed that the semivariogram of the MTVI image with $c=0.7$ increases with distance more rapidly than all the others. Comparing Figs 11 and 13, one can see that the MTVI image of this $c$ value has the biggest standard deviation. It can also be observed that as long as the standard deviation in Fig. 11 decreases, the values of the respecting semivariogram function also decrease. The same relation between standard deviation and spatial variation has also been observed in the case of an other vegetation index which was applied on a satellite image over a different area (Skianis et al. 2009). This remark indicates, without proving, that a high standard deviation of the image histogram corresponds to a strong spatial variation of the tonality of the image. If this is correct, then the probabilistic approach, although it is centered on the image histogram, may also provide information about the spatial variation of the image tonality. A further experimentation with satellite images of different areas, possibly combined with theoretical considerations, could be the subject of a future paper.

\section{Conclusions}

According to the mathematical analysis and the experimentation with the satellite image, two main conclusions can be drawn:

The standard deviation of the MTVI image depends on the ratio $\lambda / c^{2}$. For a certain value of $c$, which does not differ much from $\lambda$, the standard deviation and spatial variation of the values of the MTVI image become maximum and the tonality contrast appears strong.

Changing $c$, MTVI images with different a different optical effect are produced. Targets that are not expressed clearly in an MTVI image with a certain $c$ may appear with a more pronounced tonality contrast in an image of another $c$ value. 
The results and conclusions of this paper may help in the photointerpretation of the satellite image, in order to map burnt areas, different land covers and related morphological features. The MTVI may also serve as a tool to estimate important biophysical parameters such as leaf area index, percentage green cover and chlorophyll content, which control the behaviour and the evolution of an ecosystem, as well as the function of the system of the earth as a whole.

\section{References}

D. Haboudane, J. R. Miller, E. Pattey, P. J. Zarco-Tejada, I. B. Strachan, 2004. Hyperspectral vegetation indices and novel algorithms for predicting green LAI of crop canopies: Modeling and validation in the context of precision agriculture. Remote Sens. Environ. 90, 337-352.

Deering, D. W., Rouse, J. W., Haas, R. H., and Schell, J. A., (1975). Measuring Forage Production of Grazing Units from Landsat MSS Data. $10^{\text {th }}$ Internatonal Symposium on Remote Sensing of Environment 2, 1169-1178.

Gitelson, A. A., 2004. Wide Dynamic Range Vegetation Index for Remote Quantification of Biophysical Characteristics of Vegetation. Journal of Plant Physiology 161, 165-173.

Goel, N. S., 1988. Models of vegetation canopy reflectance and their use in estimation of biophysical parameters from reflectance data. Remote Sens. Rev. 4, 1-212.

Jensen, J. R., 2000. Remote Sensing of the Environment. Prentice Hall. 544 pages.

Kale, M., Singh, S. and Roy, P. S., 2005: Estimation of Leaf Area Index in dry deciduous forests from IRSWiFS in Central India. International Journal of Remote Sensing 26(21), 4855-4867.

Mallinis, G., Koutsias, N., Makras, A., Karteris, M., 2004. An assessment of the Information Content of Landsat -5 TM Bands for the estimation of forest stand parameters in Kassandra peninsula. Proceedings of the $7^{\text {th }}$ Panhellenic Geographical Congress, Mytilini (Greece), October $14^{\text {th }}-17^{\text {th }} 2004$, Vol. II, 415-422.

Rouse, J. W., Haas, R. H., Schell, J.A., and Deering, D. W., 1973. Monitoring vegetation systems in the Great Plains with ERTS. $3^{\text {rd }}$ ERTS Symposium, Vol. 1, 48-62.

Silleos, N. G., Alexandridis, T. K., Gitas, I. Z., and Perakis, K., 2006. Advances made in Biomasa Estimation and Vegetation Monitoring in the last 30 years. Geocarto International 21(4), 21-28.

Skianis G. Aim., Vaiopoulos D., Nikolakopoulos K., 2009. Testing the performance of the MNDVI vegetation index. SPIE Proceedings, vol. 7472, Remote Sensing of Agriculture, Ecosystems and Hydrology XI, C. U. Neale, A. Maltese (Eds), 747204. DOI: 10.1117/12.830262.

Skianis G., Vaiopoulos D., and Nikolakopoulos K., 2007a: A Comparative Study of the Performance of the NDVI, the TVI and the SAVI Vegetation Indices over burnt areas, using probability theory and spatial analysis techniques. Proceedings of the $6^{\text {th }}$ International Workshop of the EARSeL Special Interest Group on Forest Fires, 27-29 September 2007, Thessaloniki-Greece, 142-145.

Skianis, G. Aim., Vaiopoulos, D., Nikolakopoulos, K., 2007b. A Probabilistic Approach to the Problem of Assessing the Efficiency of the Transformed Vegetation Index. Int. J. Sus. Dev. Plann. 2(4), 461480 .

Spiegel, M. R., 1977. Probability and Statistics. ESPI, Athens. 384 pages.

Vaiopoulos, D. A., Skianis, G. A., Nikolakopoulos, K., 2004 . The contribution of probability theory in assessing the efficiency of two frequently used vegetation indices. International Journal of Remote Sensing 25(20), 4219-4236. 